\title{
MmWave MIMO-OFDM with Index Modulation: A Pareto-Optimal Trade-off on Spectral-Energy Efficiency
}

\author{
Yan Yang*, Shuping Dang ${ }^{\dagger}$, Miaowen Wen ${ }^{\ddagger}$, and Mohsen Guizani ${ }^{\S}$ \\ * State Key Lab. of Rail Traffic Control and Safety, Beijing Jiaotong University, Beijing, China \\ ${ }^{\dagger}$ Computer, Electrical and Mathematical Science and Engineering Division, \\ King Abdullah University of Science and Technology, Thuwal, Kingdom of Saudi Arabia \\ ${ }^{\ddagger}$ School of Electronic and Information Engineering, South China University of Technology, Guangzhou, China

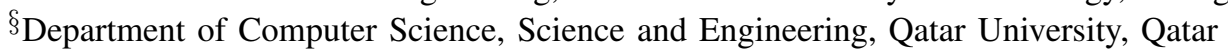 \\ *yyang@bjtu.edu.cn; ${ }^{\dagger}$ shuping.dang@kaust.edu.sa; ${ }^{\ddagger}$ eemwwen@scut.edu.cn; ${ }^{\S}$ mguizani@ieee.org
}

\begin{abstract}
Multiple-input multiple-output orthogonal frequency division multiplexing with index modulation (MIMO-OFDM-IM) has the potential advantage to balance the trade-off between spectral efficiency (SE) and energy efficiency (EE). This paper investigates the application of MIMO-OFDM-IM to millimeter wave (mmWave) communication systems. Taking advantage of the properties of Pareto optimality, we propose a feasible solution to achieve a globally Pareto-optimal trade-off between SE and EE, and the collision constraints of multi-objective optimization problem (MOP) can be solved efficiently. The MOP of SE-EE trade-off can then be converted into a Paretooptimal set (POS) solution problem. This combinatorialoriented resource allocation approach on SE-EE relation considers the optimal beam design and power reallocation for downlink multi-user mmWave transmission. We adopt the Poisson point process (PPP) to model the mobile data traffic, and the evolutionary algorithm is applied to speed up the search efficiency of the Pareto front. Compared with benchmarks, the experimental results collected from extensive simulations reveal that the proposed optimization approach is vastly superior to existing algorithms.
\end{abstract}

Keywords-MIMO-OFDM, index modulation, spectral efficiency, energy efficiency, Pareto optimal set, mmWave communication.

\section{INTRODUCTION}

Multiple-input multiple-output (MIMO) millimeter wave (mmWave) communication has aroused great expectations on enabling unprecedented high-rate transmission up to multi-gigabit for future wireless communication networks [1], [2]. To get around high path loss and severe signal attenuation, the key is the use of massiveantenna architectures at base stations (BS) and the exploitation of highly directional beamforming at both transmitter and receiver, e.g., a two-stage hybrid analogydigital (HAD) beamforming architecture. For wideband mmWave communications, the OFDM modulation can be employed to effectively combat the channel's frequency selectivity, as well as to provide further improvement in spectral efficiency (SE) [3].
As a matter of fact, the above-mentioned sophisticated technologies that require a large number of phase shifters usually consume a large amount of power, which becomes an obvious drawback. Seriously, the radio network itself could be the most energy-consuming part, and wireless operators resort to green wireless networks, where energy efficiency (EE) and SE are the main performance metrics. Unfortunately, according to the Shannon-Hartley theorem, conflicts of objects are usually difficult to balance while optimizing both SE and EE simultaneously [4]-[7]. In an effort to relax the paradox in the SEEE trade-off, an alternative way is to decompose the MOP into a number of subproblems and optimizes them simultaneously. Recently, the use of Pareto property has recently emerged as an attractive solution [8], [9].

As a novel digital modulation scheme with high SE and $\mathrm{EE}$, index modulation uses the indices of the building blocks to implicitly convey additional information bits [10]. MIMO-OFDM with index modulation (MIMOOFDM-IM) provides a beneficial transmission paradigm. The study demonstrated that MIMO-OFDM-IM can offer significantly improved transmission rates, as well as a better error performance than conventional MIMOOFDM. It has the potential to provide a flexible trade-off between SE and EE by adjusting the number of active subcarriers in each OFDM-IM subblock [11].

Motivated by these facts, we propose an SE-EE maximization MIMO-OFDM-IM scheme for downlink multiuser mmWave systems, and propose a Pareto-optimal beam design scheme for energy-efficient resource usage. By the construction of the Pareto-optimal set (POS), we propose a feasible combinatorial-oriented beam management approach to achieve a Pareto-optimal trade-off between SE and EE.

The rest of the paper is as follows. In Section II, we present the system model and a preliminary analysis of SE and EE. In Section III, we mathematically formulate the proposed Pareto-optima SE-EE trade-off and beam design. Section IV present simulation results and Section $\mathrm{V}$ conclusions of this work. 


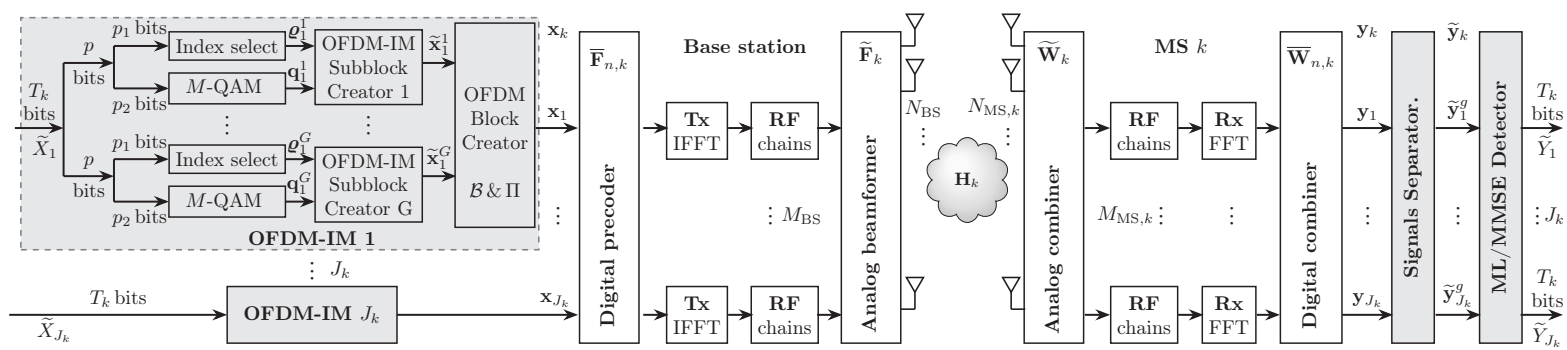

Fig. 1: Block diagram of the mmWave MIMO-OFDM-IM transceiver that employs the HAD beaforming architecture.

\section{System Model AND PROBlem Description}

\section{A. Transmission Model of mmWave MIMO-OFDM-IM}

The transceiver block diagram of MIMO-OFDM-IM for the downlink multi-user mmWave system is illustrated in Fig. 1. A BS with $N_{\mathrm{BS}}$ transmit antennas and $M_{\mathrm{BS}}$ RF chains serves $K$ active users, each of them using $N_{\mathrm{MS}, k}$ receive antennas and $M_{\mathrm{MS}, k} \mathrm{RF}$ chains. For any user $k$, we assume that the BS transmits $J_{k} \leq N_{\mathrm{MS}, k}$ data streams with $M_{\mathrm{BS}, k} \mathrm{RF}$ chains and $N_{\mathrm{BS}, k}$ transmit antennas. The HAD beamforming architecture of $\mathrm{BS}$ is constructed by the concatenation of a digital precoder $\mathbf{F}_{n, k} \in \mathbb{C}^{M_{\mathrm{BS}, k} \times N_{\mathrm{BS}, k}}$ and an analog beanformer $\widetilde{\mathbf{F}}_{k} \in \mathbb{C}^{M_{\mathrm{BS}, k} \times J_{k}}$ with a fully-connected structure. We suppose that all users have the same structure, i.e., an RF combiner $\widetilde{\mathbf{W}}_{k} \in \mathbb{C}^{J_{k} \times M_{\mathrm{MS}, k}}$ followed by a mapped digital baseband combiner $\overline{\mathbf{W}}_{n, k} \in \mathbb{C}^{N_{\mathrm{MS}, k} \times M_{\mathrm{MS}, k}}$.

Within the OFDM-IM modulator, the incoming $T_{k}$ bit stream is equally divided into $G$ groups, in which $p=T_{k} / G$ bits for each group are split into the index selection and $M$-ary modulation subgroups. For each subblock $g$, considering the number of active subcarriers $N_{A}^{k}$, the index selection subgroup contains $p_{1}$ bits for determining the indices of the active subcarriers; The $M$ ary modulation subgroup contains the remaining $p_{2}$ bits, which are mapped onto a predefined $M$-ary quadrature amplitude modulation ( $M$-QAM) signal constellation $\mathcal{S}$ to obtain the first-stage modulation subblock. We denote $\mathfrak{N}_{k}$ as the index pattern of user $k$. Thereafter, each modulated subblock is fed into a concatenated OFDM block creator to construct the main OFDM-IM blocks. For the output of the $k$ th user's data stream vector $\mathbf{x}_{j_{k}}=\left[x_{j_{k}}(1) \ldots x_{j_{k}}(N)\right]^{T}$ is independently modified by $\overline{\mathbf{F}}_{k} \in \mathbb{C}^{N_{\mathrm{BS}, k} \times N_{\mathrm{MS}, k}}$ for subsequent IFFT operation.

At the input of the OFDM index modulators, the modulated symbol can be expressed as an $T_{k} \times J_{k}$ matrix

$$
\widetilde{\mathbf{X}}_{k}=\left[\begin{array}{llll}
\widetilde{X}_{1} & \widetilde{X}_{2} & \ldots & \widetilde{X}_{J_{k}}
\end{array}\right]
$$

where $\tilde{X}_{j_{k}}=\left[\widetilde{x}_{1}, \widetilde{x}_{2}, \ldots, \widetilde{x}_{T_{k}}\right]^{T}$ is the vector of the modulated symbols at the $j_{k}$ th input of the index select and the $M$-ary modulator. $N_{A}^{k}$ active subcarriers are selected by the index selector, and the the indices of the active subcarriers can be denoted as $\varrho_{j_{k}}^{g}=\left[\begin{array}{llll}\varrho_{j_{k}}^{g}(1) & \varrho_{j_{k}}^{g}(2) & \ldots & \varrho_{j_{k}}^{g}\left(N_{A}^{k}\right)\end{array}\right]^{T}, g=$ $1,2, \ldots, G$. Meanwhile, $M$-QAM signal constellation with element $q_{j}^{g}\left(n_{A}\right) \in \mathcal{S}$ can be obtained $\boldsymbol{q}_{j_{k}}^{g}=$ $\left[q_{j_{k}}^{g}(1) q_{j_{k}}^{g}(2) \ldots q_{j_{k}}^{g}\left(N_{A}^{k}\right)\right]^{T}, g=1,2, \ldots, G$. For the $j_{k}$ th data steam, the $g$ th OFDM-IM subblock element with $p=p_{1}+p_{2}$ bits, is used to form OFDM-IM subblocks:

$$
\widetilde{\mathbf{x}}_{j_{k}}^{g}=\left[\widetilde{x}_{j_{k}}^{g}(1) \widetilde{x}_{j_{k}}^{g}(2) \ldots \widetilde{x}_{j_{k}}^{g}(N)\right]^{T}, g=1,2, \ldots, G .
$$

With the OFDM block creator $\mathcal{B}$ and interleaver $\Pi$, the transmitted FFT components for users are given by

$$
\mathbf{x}_{k}=\left[\begin{array}{llll}
\mathbf{x}_{1} & \mathbf{x}_{2} & \ldots & \mathbf{x}_{N}
\end{array}\right]^{T}, \mathbf{x}_{n} \in\{0, \mathcal{S}\}
$$

where $\mathbf{x}_{n}=\left[x_{1}, \ldots, x_{J_{k}}\right], n=1,2, \ldots, N$ is the unitpower transmitted OFDM symbol at the $j$ antenna for the $k$ th user. Suppose $P_{\mathrm{BS}, k}$ is the transmitters total power. Then the output of $\mathcal{B}$ is followed by $N \times J_{k}$ block interleavers $\Pi$ for traventional IFFT operation.

Generally, a straightforward solution to the ML detection of MIMO-OFDM-IM can be represented by

$$
\hat{\widetilde{\mathbf{x}}}_{j_{k}}^{g}=\underset{\widetilde{\mathbf{x}}_{j_{k}}^{g}}{\arg \max }\left\|\widetilde{\mathbf{y}}_{j_{k}}^{g}-\sqrt{\frac{\gamma_{k}}{N_{\mathrm{BS}, k}}} \sqrt{\frac{N}{N_{A}^{k}}} \boldsymbol{\Phi}_{j_{k}} \widetilde{\mathbf{x}}_{j_{k}}^{g}\right\|^{2} .
$$

where $\boldsymbol{\Phi}_{j_{k}}$ is an $N_{\mathrm{BS}, k} \times 1$ channel gain vector with zero-mean and unit variance, and $\gamma_{k}$ denotes the signalto-noise-ratio (SNR) per receive antenna.

Let $\widetilde{Y}_{j_{k}}=\left\{\widetilde{y}_{1}, \widetilde{y}_{2}, \ldots, \widetilde{y}_{j_{k}}\right\}$ and $\mathcal{I}\left(\widetilde{\widetilde{X}}_{j_{k}} ; \widetilde{Y}_{j_{k}}\right)$ be the output and the mutual information between $X_{j_{k}}$ and $Y_{j_{k}}$, respectively. The channel capacity $\zeta_{j_{k}}$ is given by

$$
\zeta_{j_{k}}=\sup _{x_{j_{k}}} \mathcal{I}\left(X_{j_{k}} ; Y_{j_{k}}\right)
$$

\section{B. Spectrum Efficiency and Energy Efficiency}

Without loss of generality, the composite MIMO channel between BS and user $k$ can be expressed as [12]

$$
\mathbf{H}_{k}=\sqrt{\frac{N_{\mathrm{BS}, k} N_{\mathrm{MS}, k}}{\rho}} \sum_{\ell=1}^{L} \kappa_{k}^{\ell} \mathbf{a}_{\mathrm{R}}\left(\theta_{k}^{\ell}\right) \mathbf{a}_{\mathrm{T}}^{H}\left(\phi_{k}^{\ell}\right),
$$

where $\kappa_{k}^{\ell}$ denotes the complex channel coefficient of the $\ell$ th propagation path; $\mathbf{a}_{\mathrm{T}}$ and $\mathbf{a}_{\mathrm{R}}$ is the array steering vectors, respectively. In our work, the mmWave channel estimation is simplified to estimate the channel gains, the angle-of-arrivals (AoAs) $\theta$, and angle-of-departures 
(AoDs) $\phi$ of the propagation paths. The response of the array associated with user $k$ can be modeled as

$$
\begin{aligned}
\mathbf{a}_{\mathrm{R}}\left(\theta_{k}^{\ell}\right) & =\frac{1}{\sqrt{N_{\mathrm{MS}, k}}}\left[1, e^{\cos \left(\theta_{k}^{\ell}\right)}, \ldots, e^{\left(N_{\mathrm{MS}, k}-1\right) \cos \left(\theta_{k}^{\ell}\right)}\right]^{T}, \\
\mathbf{a}_{\mathrm{T}}\left(\phi_{k}^{\ell}\right) & =\frac{1}{\sqrt{N_{\mathrm{BS}, k}}}\left[1, e^{\cos \left(\phi_{k}^{\ell}\right)}, \ldots, e^{\left(N_{\mathrm{BS}, k}-1\right) \cos \left(\phi_{k}^{\ell}\right)}\right]^{T} .
\end{aligned}
$$

From the view of implementation in the frequency domain, we define the transmitted data symbols over the $n$th subcarrier as $\mathbf{x}_{n, k} \in \mathbb{C}^{J_{k} \times 1}, n=1, \ldots, N$. The received MIMO-OFDM-IM signal of user $k$ over the $n$th subcarrier in the frequency domain is given by [2], [3]

$$
\begin{aligned}
\mathbf{y}_{n, k} & =\overbrace{\mathbf{V}_{n, k}^{H} \mathbf{H}_{k} \mathbf{U}_{n, k} \mathbf{x}_{n, k}}^{\text {primitive signal }}+\underbrace{\sum_{j \neq k} \mathbf{V}_{n, j}^{H} \mathbf{H}_{k} \mathbf{U}_{n, j} \mathbf{x}_{n, j}}_{\text {interference component }} \\
& +\mathbf{V}_{n, k}^{H} \mathbf{z}_{n, k}, n \in \mathfrak{N}_{k},
\end{aligned}
$$

where $\mathbf{V}_{n, k}=\overline{\mathbf{W}}_{k} \widetilde{\mathbf{W}}_{n, k} ; \mathbf{U}_{n, k}=\overline{\mathbf{F}}_{k} \widetilde{\mathbf{F}}_{n, k} ; \mathbf{I}_{n, j}=$ $\mathbf{V}_{n, j}^{H} \mathbf{H}_{k} \mathbf{U}_{n, j} \mathbf{x}_{n, j}$ is the interference component from other users; $\mathbf{z}_{n, k} \sim \mathcal{C N}\left(0, \sigma_{k}^{2}\right)$ is the circularly symmetric complex additive white Gaussian noise (AWGN) with mean zero and variance $\sigma_{k}^{2}$.

The received signal-to-interference-plus-noise ratio (SINR) at the $k$ th user can be represented by

$$
\operatorname{SINR}_{k}=\frac{\sum_{n \in \mathfrak{N}_{k}}\left|\mathbf{V}_{n, k}^{H} \mathbf{H}_{k} \mathbf{U}_{n, k} \mathbf{x}_{n, k}\right|^{2}}{\sum_{j \neq k} \sum_{n \in \mathfrak{N}_{j}}\left|\mathbf{V}_{n, j}^{H} \mathbf{H}_{k} \mathbf{U}_{n, j} \mathbf{x}_{n, j}\right|^{2}+\sum_{n \in \mathfrak{N}_{k}}\left|\mathbf{V}_{n, k}^{H} \mathbf{z}_{n, k}\right|^{2}} .
$$

We directly apply Theorem 1 proven in [13] to mmWave MIMO-OFDM-IM systems. The SE of user $k$ based on the Shannon formula can be written as

$$
\zeta_{k}=J_{k}\left[\begin{array}{l}
\mathbb{E}\left(\frac{N_{A}^{k}}{N}\right) \log _{2} \operatorname{det}\left(\mathbf{I}_{J_{k}}+\right. \\
\left.\mathbf{V}_{n, k} \boldsymbol{\Omega}_{n, k}^{-1} \mathbf{V}_{n, k}^{H} \mathbf{H}_{k} \mathbf{U}_{n, k}^{H} \mathbf{U}_{n, k} \mathbf{H}_{k}^{H}\right) \\
+\frac{1}{N} \log _{2} C\left(N, N_{A}^{k}\right)
\end{array}\right],
$$

where $\boldsymbol{\Omega}_{n, k}^{-1}=\mathbf{V}_{n, k}^{H} \mathbf{H}_{k}\left(\sum_{j \neq k} \mathbf{U}_{n, k} \mathbf{U}_{n, k}^{H}\right) \mathbf{H}_{k}^{H} \mathbf{V}_{n, k}+$ $\sigma^{2} \mathbf{V}_{n, k}^{H} \mathbf{V}_{n, k}$. For the effect of the number of active OFDM subcarrier and the OFDM sub-group size, we refer the interested readers to [11], [13].

The total SE in bits/s/Hz of an arbitrary user can be found by maximizing the following objective function with the constraint of transmitted power:

$$
\zeta_{\text {total }}=\sum_{k=1}^{K} \omega_{k} \zeta_{k}
$$

where $\omega_{k}$ is the weight factor for user $k ; \zeta_{k}=\sum_{j_{k}} \zeta_{j_{k}}$ is the $\mathrm{SE}$ of $k$ th user. We can determine the weights according to user's traffic types, fairness, and other priority requirements.

Generally, the power consumption model at the BS consists static and dynamic power consumption. We adopt a linear power consumption model proposed by:

$$
P_{\mathrm{sys}, k}=P_{\mathrm{BS}, k}+J_{k} P_{\mathrm{RF}, k}+P_{c, k}+N_{\mathrm{BS}, k} P_{\text {shift }},
$$

where $P_{\text {sys }, k}$ is the system power dissipation of downlink transmission of user $k ; P_{\mathrm{RF}, k}$ and $P_{\text {shift denote }}$ the power consumption of a single $\mathrm{RF}$ chain and a phase shifter, respectively; $P_{c, k}$ is the other power consumption of circuitry components. The transmitter's total power constraint $P_{\mathrm{BS}, k}$ is enforced by normalizing $\mathbf{U}_{k}$, i.e., $\sum_{n=1}^{N}\left\|\mathbf{U}_{n, k}\right\|^{2}, n \in \mathfrak{N}_{k}$. For a set of SINRs $\left\{\operatorname{SINR}_{k}\right\}_{k=1}^{K}$, the total SINR scales in a multi-user system can be represented by

$$
\operatorname{SINR}_{\text {sys }}=\sum_{k=1}^{K} \omega_{k} \operatorname{SINR}_{k} .
$$

To guarantee the fairness among users in the multiuser network, the max-min SINR problem is considered, which deals with the sum-rate maximization problem and guarantees the worst performance of receivers. Typically, the max-min SINR problem satisfying the overall transmit power constraint can be formulated as

$$
\begin{aligned}
& \begin{array}{lll}
\underset{\left\{\mathbf{U}_{k}, \mathbf{V}_{k}, \forall k\right\}}{\max } & \min & \operatorname{SINR}_{k}\left(P_{\mathrm{BS}, k}\right), \\
\text { s.t. } & & P_{\mathrm{BS}, k} \leq P_{\mathrm{BS}, k}^{\max }, \quad \forall k \\
& &
\end{array} \\
& K \leq \sum_{k=1}^{K} J_{k} \leq M_{t} \text {, }
\end{aligned}
$$

where $P_{\mathrm{BS}, k}^{\max }$ is the maximum transmission power.

The objective function in terms of EE is defined as the system capacity divided by the total power consumption, i.e., the transmitted information bits per unit energy in the unit of bits per Joule. The EE (bits/J) of user $k$ with bandwidth $B_{T_{k}}$ is then defined as

$$
\begin{array}{cl}
\max _{\left\{\mathbf{U}_{k}, \mathbf{V}_{k}, \forall k\right\}} & \eta_{k}=\frac{B_{T_{k}} \cdot \zeta_{k}}{P_{\mathrm{BS}, k}+J_{k} P_{\mathrm{RF}, k}+P_{c, k}+N_{\mathrm{BS}, k} P_{\mathrm{shift}}} \\
\text { s.t. } & P_{c, k}=\sum_{n=1}^{N}\left\|\mathbf{U}_{n, k}\right\|^{2} \leq P_{\mathrm{BS}, k}^{\max } .
\end{array}
$$

According to the classical SE-EE trade-off paradigm, the objective function can be rewritten

$$
\eta_{\text {total }}=\frac{B_{T} \cdot \zeta_{\text {total }}}{\log _{2}\left(\sum_{k=1}^{K} P_{\mathrm{sys}, k}\right)} .
$$

\section{PARETO-OPTIMAL TRADE-OFF ON SPECTRAL-ENERGY EFFICIENCY}

\section{A. Fundamental Limitations of MOP}

Generally, a maximization MOP with $\mathfrak{m}$ objective functions can be formulated as follows:

$$
\begin{array}{ll}
\operatorname{maximize} & F(\chi)=\left(f_{1}(\chi), \ldots, f_{\mathfrak{m}}(\chi)\right)^{T}, \\
\text { s.t. } & \chi \in \mathcal{X},
\end{array}
$$

where $F(\chi)$ is a vector function, that is, each element is an objective function; $\chi=\left\{\chi_{1}, \ldots, \chi_{\mathfrak{n}}\right\}$ is a feasible variable set selected from the decision variable space, aiming to maximize each objective function by searching to identify an optimal solution. Let $\mathcal{F}$ and $\mathcal{X}$ be the objective function space and decision variable space, respectively. The decision space represented by MOP can be characterized by tuple $\mathcal{M}=(\mathcal{F}, \mathcal{X})$. 


\section{B. Pareto-Optimal Solution to SE-EE Trade-off}

To ease expositions, we use $f_{\mathrm{SE}}(\cdot), f_{\mathrm{EE}}(\cdot), f_{\mathrm{SINR}}(\cdot)$ to represent the objective functions with respect to $\mathrm{SE}, \mathrm{EE}$ and SINR, respectively. For the $k$ th user, the objective function can be expressed as

$$
F_{k}\left(\chi_{k}\right)=\left(f_{\mathrm{SE}}\left(\mu_{k}\right), f_{\mathrm{EE}}\left(\nu_{k}\right), f_{\mathrm{SINR}}\left(\xi_{k}\right)\right), F_{k} \in \mathcal{F},
$$

where $\mu_{k}, \nu_{k}, \xi_{k} \in \mathcal{X}$ is the set of the decision vectors with the same or different variable size(s).

To achieve the global SE-EE optimality, our solution resorts to the decomposition of the optimization process. First, SE and EE maximization of every user is performed in a best-efford fashion without setting other priority balance, whilst SINR is approximately constant. Using this problem transformation, the Pareto-optimal SE and EE is transformed into a design problem of the optimal beam and the paradox in (18) can be relaxed by sequentially solving the following optimization problem:

$$
\begin{array}{ll}
\max & f_{\mathrm{SE}}\left(\mu_{k}\right)=f_{\mathrm{SE}}\left(\mathbf{U}_{k}, \mathbf{V}_{k}, \mathbf{P}_{k}, J_{k}\right) \\
\max & f_{\mathrm{EE}}\left(\boldsymbol{v}_{k}\right)=f_{\mathrm{EE}}\left(\mathbf{U}_{k}, \mathbf{V}_{k}, \mathbf{P}_{k}, J_{k}\right) \\
\text { s.t. } & \sum_{k=1}^{K} J_{k} \leq M_{t}, \\
& \sum_{k=1}^{K} P_{\mathrm{BS}, k} \leq \sum_{k=1}^{K} P_{\mathrm{BS}, k}^{\max },
\end{array}
$$

and

$$
\begin{array}{lc}
\max _{\overline{\boldsymbol{\alpha}}} \min _{\mathbf{P}_{k}, J_{k}} & f_{\mathrm{SINR}}\left(\xi_{k}\right)=\sum_{k=1}^{K} \bar{\alpha}_{k} f_{\mathrm{SINR}}\left(\mathbf{P}_{k}, J_{k}\right) \\
\text { s.t. } & \sum_{k=1}^{K} \bar{\alpha}_{k}=1,
\end{array}
$$

where $\overline{\boldsymbol{\alpha}}^{T} \in \mathbf{R}^{1 \times K}$ with rows $\alpha_{k} \in[0,1] ; \mu, \nu$ and $\xi$ are constructed by those limited variable combinations that have a significant impact on the objective function; $\mathbf{P}_{k}=\left(P_{1}, \ldots, P_{J_{k}}\right)$ represents the link power allocation outcome. The key to solve (19) and (20) is to find the optimal POS from the feasible decision vectors. The feasible set $\mathcal{X}$ is a linear combination of all decision vectors. For $k$ th user, it can be given by $\mathcal{X}: \mathcal{X}_{k}=$ $\left\{\mathbf{U}_{k}, \mathbf{V}_{k}, \mathbf{P}_{k}, J_{k}\right\}, \forall k$. We refer to this strategy as a combinatorial-oriented beam design approach.

In particular, high directivity beam is dominated by the phased array. This fact in turn implies that the aligned beam pairs are always Pareto-optimal. The design of Pareto-optimal beam can be cast as the optimization problem on digital parts, i.e., $\widetilde{\mathbf{F}}_{k}$. and $\widetilde{\mathbf{W}}_{k}$ can be independently optimize. $\widetilde{\mathbf{F}}_{k}^{\star}$ and $\widetilde{\mathbf{W}}_{k}^{\star}$ can be found by assuming that $\overline{\mathbf{F}}_{k}$ and $\overline{\mathbf{W}}_{k}$ are fixed. Based on (8), the $\mathbf{U}_{k}$ with local optimality at the BS can be thereby determined by jointly solving the following optimization problem:

$$
\begin{aligned}
& \widetilde{\mathbf{F}}_{n, k}^{\star}=\underset{\widetilde{\mathbf{U}}_{n, k}, \forall k}{\arg \max } \quad \mathbf{U}_{n, k}^{H} \mathbf{x}_{n, k}^{H} \mathbf{x}_{n, k} \mathbf{U}_{n, k} \\
& \text { s.t. } \quad \sum_{n=1}^{N} \operatorname{Tr}\left(\mathbf{U}^{H}{ }_{n, k} \mathbf{U}_{n, k}\right)<P_{\mathrm{BS}, k},
\end{aligned}
$$

At the receiver, the feasilble $\mathbf{V}_{k}^{\star}$ associated with $\tilde{\mathbf{V}}_{k}^{*}$ for each user can be computed in the same way as

$$
\begin{array}{cc}
\widetilde{\mathbf{W}}_{n, k}^{\star}=\underset{\widetilde{\mathbf{W}}_{n, k}, \forall k}{\arg \max } & \frac{\left|\mathbf{V}_{n, k}^{H} \mathbf{H}[k] \mathbf{U}_{n, k}\right|^{2}\left|\mathbf{x}_{n, k}\right|^{2}}{\left|\mathbf{V}_{n, k}^{H} \mathbf{z}_{n, k}\right|^{2}} \\
\text { s.t. } & \sum_{n=1}^{N}\left|\mathbf{V}_{n, k}\right|^{2}=1 .
\end{array}
$$

By removing $\widetilde{\mathbf{F}}_{k}$ and $\widetilde{\mathbf{W}}_{k}$ from the problem formulated in (19) for the $k$ th user, the problem of finding a set of decision vectors $\mathcal{X}_{k}^{\star}$ that maximizes the $\mathrm{SE}$ and EE can be represented by

$$
\max _{\mathcal{X}_{k}^{\star}, \forall k}\left(f_{\mathrm{SE}}\left(\overline{\mathbf{F}}_{k}, \overline{\mathbf{W}}_{k}, \mathbf{P}_{k}, J_{k}\right), f_{\mathrm{EE}}\left(\overline{\mathbf{F}}_{k}, \overline{\mathbf{W}}_{k}, \mathbf{P}_{k}, J_{k}\right)\right.
$$

To generate universally Pareto-optimal SE-EE maximization, we apply a weighted-sum criterion to capture multiple objectives, where multiple metrics are scalarized into a single-objective function. This linear weighted-sum method can be expressed as

$$
\begin{array}{ll}
\arg \max _{\mathcal{X}, \overline{\boldsymbol{\alpha}}} & \sum_{k=1}^{K} \bar{\alpha}_{k} F\left(\mu_{k}, \nu_{k}, \xi_{k}\right) \\
\text { s.t. } & \mu_{k}, \nu_{k}, \xi_{k} \in \mathcal{X}^{\star}, \\
& \sum_{k=1}^{K} \bar{\alpha}_{k}=1,
\end{array}
$$

where $\overline{\boldsymbol{\alpha}}^{T} \in \mathbf{R}^{K \times 1}$ with rows $\bar{\alpha}_{k}$.

Once the objective values of $\mathcal{X}^{\mathrm{POS}}$ with respect to the Pareto optimality are achieved. It means that $\mathcal{X}^{\text {POS }}$ is not dominated by any other feasible decision vectors and satisfies $F\left(\mathcal{X}^{\mathrm{POS}}\right) \succeq F\left(\mathcal{X}^{\star}\right)$, with objective set $\left\{\overline{\mathbf{F}}_{k}^{\mathrm{POS}}, \overline{\mathbf{W}}_{k}^{\mathrm{POS}}, \mathbf{P}_{k}^{\mathrm{POS}}, J_{k}^{\mathrm{POS}}\right\}$. The objective value of a set of Pareto-optimal decision vectors constitutes the Pareto front. In this case, the feasible $\mathcal{X}^{\star}$ is spanned by linear space because the elements of objective vector set $\left\{\overline{\mathbf{F}}_{k}^{\mathrm{POS}}, \overline{\mathbf{W}}_{k}^{\mathrm{POS}}, \mathbf{P}_{k}^{\mathrm{POS}}, J_{k}^{\mathrm{POS}}\right\}$ are linear.

Next, we apply Cobb-Douglas production function to characterize the trade-off on SE-EE. It can be expressed by a utility function $U$, which has the form [14]

$$
U\left(f_{\mathrm{SE}}\left(\mathcal{X}_{k}^{\star}\right), f_{\mathrm{SE}}\left(\mathcal{X}_{k}^{\star}\right)\right)=\left(\widehat{f_{\mathrm{SE}}}\left(\mathcal{X}_{k}^{\star}\right)\right)^{\alpha_{k}}\left(\widehat{f_{\mathrm{EE}}}\left(\mathcal{X}_{k}^{\star}\right)\right)^{1-\alpha_{k}}
$$

where $\widetilde{f_{\mathrm{SE}}}\left(\mathcal{X}_{k}^{\star}\right)=\frac{f_{\mathrm{SE}}\left(\mathcal{X}_{k}^{\star}\right)}{f_{\mathrm{SE}}^{\max }\left(\mathcal{X}_{k}^{\star}\right)}$ and $\widetilde{f_{\mathrm{EE}}}\left(\mathcal{X}_{k}^{\star}\right)=\frac{f_{\mathrm{EE}}\left(\mathcal{X}_{k}^{\star}\right)}{f_{\mathrm{EE}}^{\max }\left(\mathcal{X}_{k}^{\star}\right)}$; $\alpha_{k} \in[0,1]$ is a weight metric on a trade-off between SE and $\mathrm{EE}$ for the conflicts of interest. With this empirical production function, different weights can be viewed as the benefit-cost ratio of the system. A logarithmic form of above function can be given as follows:

$$
\begin{aligned}
\mathcal{H}_{k}: \mathcal{H}\left(\mathcal{X}_{k}^{\star}\right) & =\alpha_{k} \log \left(\widehat{f_{\mathrm{SE}}}\left(\overline{\mathbf{F}}_{k}^{\star}, \overline{\mathbf{W}}_{k}^{\star}, \mathbf{P}_{k}^{\star}, J_{k}^{\star}\right)\right. \\
& +\left(1-\alpha_{k}\right) \log \widehat{f_{\mathrm{EE}}}\left(\overline{\mathbf{F}}_{k}^{\star}, \mathbf{P}_{k}^{\star}, J_{k}^{\star}\right) .
\end{aligned}
$$

Note that $\bar{\alpha}$ and the weight vector with respect to $U$ are equivalent. Then, the above MOP of finding a $\mathcal{X}^{\text {POS }}$ is transformed into a single globally optimal problem:

$$
\begin{array}{cl}
\max _{\left\{\overline{\mathbf{F}}_{k}^{\star}, \overline{\mathbf{W}}_{k}^{\star}, \mathbf{P}_{k}^{\star}, J_{k}^{\star}\right\}} & \mathcal{H}\left(\overline{\mathbf{F}}_{k}^{\star}, \overline{\mathbf{W}}_{k}^{\star}, \mathbf{P}_{k}^{\star}, J_{k}^{\star}\right), \\
\text { s.t. } & \left\{\overline{\mathbf{F}}_{k}^{\star}, \overline{\mathbf{W}}_{k}^{\star}, \mathbf{P}_{k}^{\star}, J_{k}^{\star}\right\} \in \mathcal{X}^{\star}, \\
& \sum_{k=1}^{K} J_{k}^{\star} \leq \sum_{k=1}^{K} J_{k} .
\end{array}
$$

Considering the optimal target set of the overall user in the whole beamspace, the global trade-off between SE and EE can be expressed as

$$
\begin{aligned}
\mathcal{H}_{\text {global }} \succeq & \sum_{k=1}^{K}\left(\alpha_{k} f_{\mathrm{SE}}\left(\overline{\mathbf{F}}_{k}^{\star}, \overline{\mathbf{W}}_{k}^{\star}, \mathbf{P}_{k}^{\star}, J_{k}^{\star}\right)\right. \\
& \left.+\left(1-\alpha_{k}\right) f_{\mathrm{EE}}\left(\overline{\mathbf{F}}_{k}^{\star}, \overline{\mathbf{W}}_{k}^{\star}, \mathbf{P}_{k}^{\star}, J_{k}^{\star}\right)\right),
\end{aligned}
$$


In the user-centric scenario, the over-large transmit power does not necessarily help to improve the total SE, since the interference would dramatically increase. To achieve the optimal SINR, the worst-case (weak users) and best-case (strong users) SINR optimization need to be balanced. Based on the above analysis, we propose a combinational power control strategy to handle the worst and the best cases. With the aid of MIMO-OFDM-IM, a power reallocation can be applied to balance the total transmit power. Let $\mathcal{P}=\left(\beta_{1}, \ldots, \beta_{K}\right)^{T}$ be the instantaneous power reallocation set. We thereby introduce a new combination of decision variable set $\Psi=\left\{\mathbf{P}_{k}, J_{k} ; \beta_{k}\right\}$.

Accordingly, the optimization problem of (20) can be transferred to the following optimization problem:

$$
\begin{aligned}
& \max _{\left\{\overline{\mathbf{F}}_{k}^{\star}, \overline{\mathbf{W}}_{k}^{\star}\right\}, \boldsymbol{\alpha}} \min _{\mathbf{P}_{k}^{\text {POS }}, J_{k}^{\mathrm{POS}}} \sum_{k=1}^{K} \alpha_{k} f_{\mathrm{SINR}}\left(\mathbf{P}_{k}^{\star}, J_{k}^{\star} ; \beta_{k}\right), \\
& \text { s.t. } \quad P_{\mathrm{BS}, k} \leq P_{\mathrm{BS}, k}^{\max }, \quad \forall k \text {. }
\end{aligned}
$$

In our work, the desired quantization levels of SINR is indicated by $\boldsymbol{\alpha}$ and $\mathcal{P}$, which can used to improve SINR of weak users. If all of the constraints in (29) are satisfied, the reduced $J_{k}^{\mathrm{POS}}$ and $\mathbf{P}_{k}^{\mathrm{POS}}$ are the optimal power allocation scheme. When coefficients of $\boldsymbol{\alpha}$ are properly balanced, we can achieve an approximately complete Pareto optimality, i.e., $\mathbf{P}_{k}^{\mathrm{POS}}$ and $J_{k}^{\mathrm{POS}}$. Obviously, if all of the constraints in (29) are satisfied, we can obtain the partially suppressed $J_{k}^{\mathrm{POS}}$ and the feasible power reallocation scheme $\mathbf{P}_{k}^{\mathrm{POS}}$ for all users.

With the combined decision variable set $\Psi$ with the Pareto optimality, the global optimal Pareto solution can be found by alternatingly solving the following problem:

$$
\begin{gathered}
\max _{\overline{\mathbf{F}}_{k}^{\mathrm{POS}}, \overline{\mathbf{W}}_{k}^{\mathrm{POS}}} \mathcal{H}_{\mathrm{global}} \succeq \mathcal{H}\left(\overline{\mathbf{F}}_{k}^{\star}, \overline{\mathbf{W}}_{k}^{\star}, \mathbf{P}_{k}^{\mathrm{POS}}, J_{k}^{\mathrm{POS}}, \beta_{k}\right) \\
\text { s.t. } \quad\left\{\mathbf{P}_{k}^{\mathrm{POS}}, J_{k}^{\mathrm{POS}}, \beta_{k}\right\} \in \Psi, \forall k .
\end{gathered}
$$

Since the objective function in (30) is convex, it can be iteratively solved. In this case, a set of candidates $\mathcal{X}^{\star}$ could gradually evolve to $\mathcal{X}^{\mathrm{POS}}$. For all the dominated solutions, the lower solution will be removed. We show that the solution to finding POS towards the true Pareto front can be calculated by means of a multi-objective evolutionary algorithm, non-dominated sorting genetic algorithm-II (NSGA-II) [15]. It is initialized by the initial user discovery as well as the beam training procedure. In the first stage, SE and EE maximization of each user is performed in a best-efford fashion without setting any priority balance. The joint maximization of SE and EE for each user is performed concurrently, and we thereby achieve a single locally Pareto-optimal solution. In the second stage, the global trade-off between SE and EE is taken into account. It mainly aims to apply NSGA-II for finding the desired POS under the SINR constraints.

\section{NuMERICAL RESUlTS}

We consider the case of a single-cell mmWave MIMO-OFDM-IM system at $28 \mathrm{GHz}$ frequency bands. Each user can be modeled as an independent homogeneous Poisson point process (PPP) with intensity $\lambda_{k}$, i.e.,

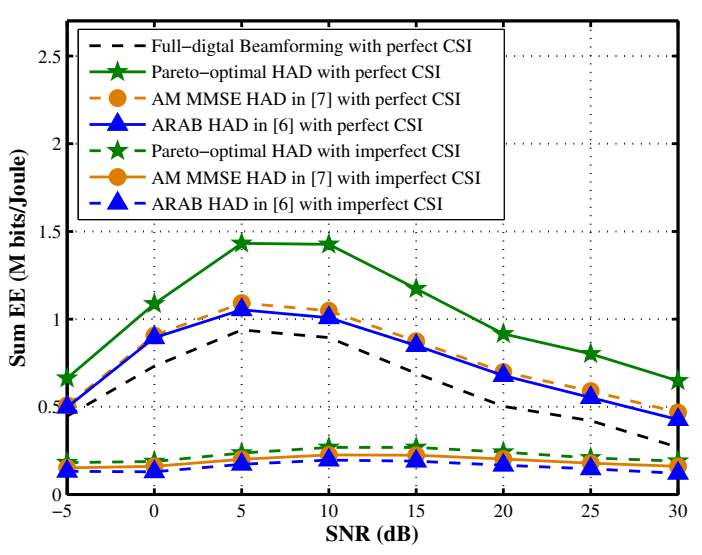

Fig. 2: Comparison of the EE performance at high SNR.

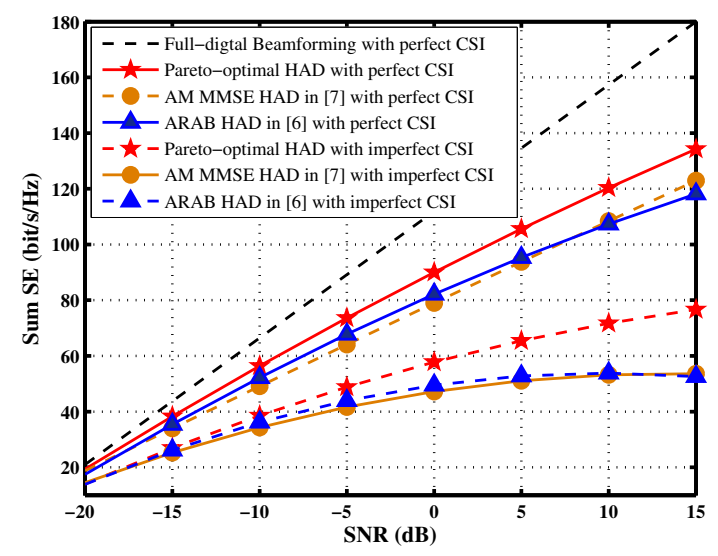

Fig. 3: Comparison of the SE performance at low SNR.

$\Gamma_{k}=\left\{\left(r_{k}, \varphi_{k} ; \lambda_{k}\right): k=1, \ldots, K\right\}$, where $\left(r_{k}, \varphi_{k}\right)$ is a two-dimensional polar coordinate. The BS with 16 $\mathrm{RF}$ chainsand MS with $4 \mathrm{RF}$ chains are equipped with $N_{\mathrm{BS}}=256, N_{\mathrm{MS}}=16$ antenna elements, respectively. In addition, we set $P_{\mathrm{RF}}=250 \mathrm{~mW}, P_{c, k}=200 \mathrm{~mW}$ and $P_{\text {shift }}=88 \mathrm{~mW}$.

In Fig. 2, we evaluate the $\mathrm{SE}$ and $\mathrm{EE}$ performance in the high SNR regime by considering the impact of perfect and imperfect CSI, respectively. We compare the proposed Pareto-optimal HAD beamforming scheme with the approximation methods proposed in [6], and in [7] that can be extended to MIMO-OFDM configurations without changing the antennas setup. It is clear that our proposed approach has a much higher EE than those of the algorithms proposed in [6], [7].

In Fig. 3 and 4, we can observe that the proposed approach with equal power allocation can significantly outperform its counterpart. That is, the proposed approach achieves higher SE and EE than those of the existing approaches designed in [6], [7]. For weak users, it is a competitive technology to provide a much higher 


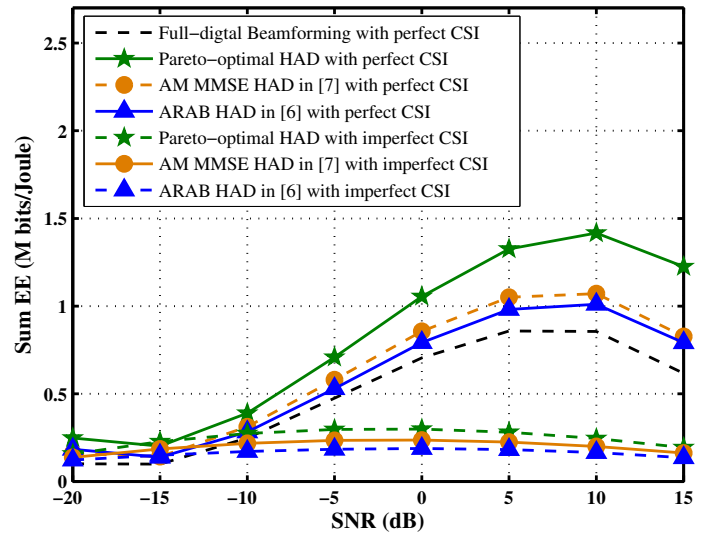

Fig. 4: Comparison of the EE performance at low SNR.
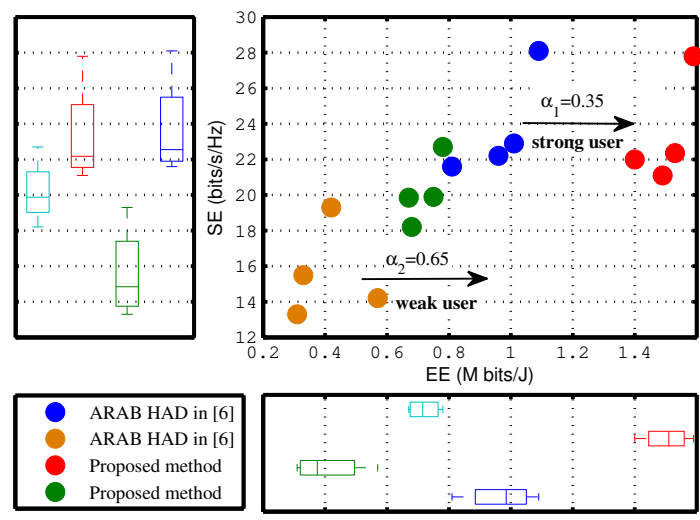

Fig. 5: A globally optimal SE-EE trade-off with POS.

data rate and a longer transmission distance, and has the ability to extend the coverage. It can be explained that the benefits of power allocation for MIMO-OFDMIM, the subcarriers with high power can be scheduled for weak users. Even with both a total transmit power constraint, the achievable sum rate in the low SNR regime is maximized by exploiting the systematic optimization.

Fig. 5 illustrates a typical case with the SE-EE tradeoff. As a case study, we consider a realistic communication scenario, which consists of four strong users and four weak users, and we aim to maximize the SE and EE, simultaneously. A key observation of Fig. 5 is that by properly adjusting weighting coefficients $\alpha$, the performance of SE and EE can be significantly improved, which fully verifies the theoretical analysis. The proposed approach is clearly superior to the one given in [6]. Once both SE and EE are maximized as far as possible, these points (possible POS), can be approximately viewed as Pareto front. For the same reasons pointed out above, with the help of OFDM-IM, it allows more degrees of freedom to achieve realistic SE-EE maximization.

\section{CONCLUSION}

In this paper, an energy-efficient mmWave MIMOOFDM-IM system was proposed. We provided the optimal solution that allows a higher degree of freedom to achieve SE-EE maximization in mmWave cellular networks, and a baseline design to solve the SE-EE tradeoff has been given. The key finding of this study is that the use of Pareto optimality can achieve a globally optimal trade-off between SE and EE, and the collision constraints of MOP can be efficiently released.

\section{ACKNOWLEDGEMENT}

This work was supported by the research task of the State Key Laboratory of Rail Traffic Control and Safety (Contract No. RCS2020ZT012), Beijing Jiaotong University, and the key research task of China Railway Corporation (Contract No. N2019G028).

\section{REFERENCES}

[1] Z. Pi and F. Khan. An introduction to millimeter-wave mobile broadband systems. IEEE Commun. Mag., 49(6):101-107, 2011.

[2] F. Sohrabi and W. Yu. Hybrid digital and analog beamforming design for large-scale antenna arrays. IEEE J. Sel. Topics Signal Process., 10(3):501-513, April 2016.

[3] A. Alkhateeb, J. Palacios, and R. W. Heath, Jr. Frequency selective hybrid precoding for limited feedback millimeter wave systems. IEEE Trans. Commun., 64:1801-1818, May 2016.

[4] A. Puglielli et al. Design of energy- and cost-efficient massive MIMO arrays. Proc. IEEE, 104(3):586-606, March 2016.

[5] L. Sboui et al. A new relation between energy efficiency and spectral efficiency in wireless communications systems. IEEE Wireless Commun., 26:168-174, June 2019.

[6] K. Ardah et al. Hybrid analog-digital beamforming design for $\mathrm{SE}$ and EE maximization in massive MIMO networks. IEEE Trans. Veh. Technol., 69(1):377-389, January 2020.

[7] J. P. González-Coma et al. Channel estimation and hybrid precoding for frequency selective multiuser mmwave MIMO systems. IEEE J. Sel. Areas Commun., 12(2):353-367, May 2018.

[8] M. D. Renzo et al. Spectral-energy efficiency pareto front in cellular networks: A stochastic geometry framework. IEEE Wireless Commun. Lett., 8(2):424-427, April 2019.

[9] R. Hu and T. Lok. Pareto optimality for the single-stream transmission in multiuser relay networks. IEEE Trans. Wireless Commun., 16(10):6503-6513, October 2017.

[10] R. A. Alhiga and H. Haas. Subcarrier-index modulation OFDM. In IEEE 20th Int. Symp. Pers., Indoor Mobile Radio Commun. (PIMRC), pages 177-181, Tokyo, Japan, September 2009.

[11] E. Basar. On multiple-input multiple-output OFDM with index modulation for next generation wireless networks. IEEE Trans. Signal Process., 64(15):3868-3878, August 2016.

[12] A. A. M. Saleh and R. Valenzuela. A statistical model for indoor multipath propagation. IEEE J. Sel. Areas Commun., 5(2):128137, February 1987.

[13] M. Wen et al. On the achievable rate of OFDM with index modulation. IEEE Trans. Signal Process., 64(8):1919-1932, April 2016.

[14] J. B. Rao and A. O. Fapojuwo. On the tradeoff between spectral efficiency and energy efficiency of homogeneous cellular networks with outage constraint. IEEE Trans. Veh. Technol., 62(4):1801-1814, May 2013.

[15] K. Deb et al. A fast and elitist multiobjective genetic algorithm: NSGA-II. IEEE Trans. Evol. Comput., 6(2):185-197, April 2002. 